\title{
Effects of dibutyryl cyclic AMP on oocyte maturation and ovulation in the perfused rabbit ovary*
}

\author{
Y. Hosoi, Y. Yoshimura, S. J. Atlas, T. Adachi and E. E. Wallach \\ The Johns Hopkins University School of Medicine, Department of Gynecology and Obstetrics, \\ 600 N. Wolfe Street, Baltimore, Maryland 21205, USA
}

\begin{abstract}
Summary. The involvement of cyclic AMP (cAMP) in mammalian oocyte maturation was assessed using cultures of rabbit cumulus-oocyte complexes and in-vitro perfused rabbit ovaries. Rabbit cumulus-oocyte complexes were cultured in Brackett's medium with or without dibutyryl cyclic AMP $\left((\mathrm{Bu})_{2} \mathrm{CAMP}\right)$ at $10^{-3}, 10^{-4}$ or $10^{-5} \mathrm{M}$ for 4-12 h. At $4 \mathrm{~h}$ spontaneous meiotic maturation was significantly inhibited by $(\mathrm{Bu})_{2}$ cAMP $(P<0.025)$. With prolonged incubation, spontaneous maturation progressed despite exposure to $(\mathrm{Bu})_{2} \mathrm{cAMP}$. When ovaries were continuously perfused in vitro for $12 \mathrm{~h}$ with $(\mathrm{Bu})_{2} \mathrm{cAMP}\left(10^{-3} \mathrm{M}\right)$ or medium alone, $(\mathrm{Bu})_{2} \mathrm{cAMP}$ stimulated ovarian progesterone production, but did not affect ovulation or maturation of follicular oocytes. When ovaries were perfused in vitro with or without $(\mathrm{Bu})_{2} \operatorname{cAMP}\left(10^{-3}\right.$, $10^{-4}$ or $10^{-5} \mathrm{M}$ ) for the first $2 \mathrm{~h}$ and then transferred to medium without $(\mathrm{Bu})_{2} \mathrm{cAMP}$ for an additional $10 \mathrm{~h}$, ovulation did not occur, but transient exposure to $(\mathrm{Bu})_{2} \mathrm{cAMP}$ stimulated a dose-related increase in maturation of follicular oocytes. Degeneration of follicle-enclosed oocytes and cumulus-oocyte complexes was not affected by exposure to $(\mathrm{Bu})_{2} \mathrm{cAMP}$. These results suggest that transient, but not continuous, elevation of cAMP after the gonadotrophin surge may be required for the initiation of oocyte maturation.
\end{abstract}

Keywords: cAMP; rabbit; oocyte maturation; ovulation

\section{Introduction}

Mammalian oocytes in non-atretic follicles are arrested at the diplotene stage of the first meiotic division until the preovulatory surge of gonadotrophins initiates resumption of meiosis (Thibault, 1977). Follicle-enclosed oocytes cultured in vitro remain immature until they are exposed to luteinizing hormone $(\mathrm{LH})$ or human chorionic gonadotrophin (hCG) (Liberman et al., 1976; Kobayashi et al., 1981). However, when oocytes are liberated from antral follicles and cultured, they resume meiotic maturation spontaneously, independent of hormonal stimulation (Pincus \& Enzmann, 1935). Exposure of isolated cumulus-oocyte complexes to membrane-permeable cAMP derivatives or phosphodiesterase inhibitors results in a reversible block of spontaneous maturation in vitro (Cho et al., 1974; Dekel \& Beers, 1978). These observations suggest that oocytes may be maintained in the dictyate state in vivo via the passage of cAMP from cumulus cells to the oocyte.

The in-vitro perfused rabbit ovary preparation has been an effective model for studying local factors involved in the ovulatory process and for evaluating the effects of specific agents on phenomena associated with oocyte maturation (Kobayashi et al., 1981; Yoshimura et al., 1985). In this study the model was used to examine the effects of cAMP on ovulation and oocyte maturation. The effects of cAMP on in-vitro maturation of rabbit oocytes were also re-examined with respect to

*Reprint requests to Dr E. E. Wallach. 
time of exposure. These experiments were designed to explore the paradoxical situation in which LH induces oocyte maturation and stimulates production of ovarian cAMP, while exposure of cumulus-oocyte complexes to cAMP inhibits spontaneous maturation.

\section{Materials and Methods}

Animals. Sexually mature New Zealand White rabbits (3.5-4.5 kg body weight) were used. Rabbits were isolated for a minimum of 3 weeks before the experimental procedure and were caged individually under controlled temperature $\left(21 \cdot 1-22 \cdot 2^{\circ} \mathrm{C}\right)$ and light $(07: 00 \mathrm{~h} \cdots 21: 00 \mathrm{~h})$ with free access to Purina rabbit chow and water. The rabbits were anaesthetized with intravenous pentobarbitone sodium $(32 \mathrm{mg} / \mathrm{kg})$, given heparin sulphate $(120 \mathrm{U} / \mathrm{kg})$ for anticoagulation, and then subjected to laparotomy.

Culture of isolated oocytes. Ovaries were removed and placed in Brackett's defined medium (Brackett \& Oliphant, 1975). Under a dissecting microscope, follicles of $>1.0 \mathrm{~mm}$ in diameter were punctured with a 26-gauge needle to release follicular contents. The cumulus-oocyte complexes were identified and washed 3 times in fresh medium, and then cultured in $1.0 \mathrm{ml}$ medium at $37^{\circ} \mathrm{C}$ and $100 \%$ humidity using $5 \% \mathrm{CO}_{2}$ in air for $4-12 \mathrm{~h}$.

Ovarian perfusion. Each ovarian artery was cannulated in situ, and the ovary and its vascular pedicle were removed and placed in the perfusion chamber. Each ovary was perfused for $12 \mathrm{~h}$ with $150 \mathrm{ml}$ tissue culture Medium 199 (M.A. Bioproducts, Walkerville, MD, USA) supplemented with insulin (20 U/litre; Squibb-Nova, Inc., Princeton, NJ, USA), heparin sulphate (200 U/litre; Invenex Laboratories, Chagrin Falls, OH, USA), streptomycin $(50 \mathrm{mg} / \mathrm{litre}$; Sigma Chemical Co., St Louis, MO, USA), and penicillin G $(75 \mathrm{mg} / \mathrm{litre}$; Sigma), and adjusted to $\mathrm{pH} 7 \cdot 4$. The cannulation procedure and perfusion apparatus have been previously described in detail (Lambertsen et al., 1976; Kobayashi et al., 1981). Ovaries were periodically observed for evidence of follicle growth and rupture. At $12 \mathrm{~h}$ after the onset of perfusion, follicular oocytes were recovered from mature follicles ( $>1.5 \mathrm{~mm}$ in diameter), and the experiment was concluded. Oocytes were assessed for the stage of maturity and signs of degeneration.

Examination of oocytes. At the conclusion of either culture or perfusion, cumulus cells were removed from oocytes with a solution of $0.5 \mathrm{mg}$ hyaluronidase $/ \mathrm{ml}$ (Sigma) and gentle agitation with a narrow-bore pipette. Oocytes were placed on slides, fixed in $2.5 \%$ glutaraldehyde and then stained with $0.25 \%$ lacmoid in $45 \%$ acetic acid for microscopic evaluation. Oocytes were assessed for stage of maturity as previously described (Kobayashi et al., 1981). The degree of oocyte maturity was expressed as the percentage of oocytes which had achieved germinal vesicle breakdown (GVBD). Oocytes were also assessed for degenerative changes, including vacuolation, cytolysis, necrosis and loss of spherical shape.

Experiment 1 . The initial experiment was designed to determine whether cAMP inhibits spontaneous maturation of cumulus-oocyte complexes removed from rabbit ovarian follicles. The complexes recovered from rabbit ovaries were cultured in Brackett's medium with or without $(\mathrm{Bu})_{2} \mathrm{CAMP}$ (Sigma) at a concentration of $10^{-5}, 10^{-4}$ or $10^{-3} \mathrm{M}$. The maturity of individual oocytes was evaluated after 4,6 or $12 \mathrm{~h}$ of culture.

Experiment II. This experiment was designed to determine whether cAMP plays a significant role in the process of follicle rupture. $(\mathrm{Bu})_{2} \mathrm{cAMP}$ at a concentration of $10^{-3} \mathrm{M}$ was added to the perfusate of 1 ovary from each of the 6 rabbits. The contralateral ovary of each rabbit was simultaneously perfused in a separate chamber with medium alone and served as a control. Both ovaries were perfused for a total of $12 \mathrm{~h}$. Perfusate samples $(3 \mathrm{ml})$ were withdrawn at the onset of perfusion and at $1,2,4,6,8$ and $12 \mathrm{~h}$ therafter and were immediately replaced by an equal volume of fresh medium. The samples were stored at $-20^{\circ} \mathrm{C}$ until measurement of progesterone by radioimmunoassay (RIA). In an additional group of 6 rabbits, progesterone concentration was also determined in samples of perfusate collected after exposure to hCG (50 i.u.) in order to compare progesterone values with those observed in $(\mathrm{Bu})_{2} \mathrm{cAMP}$-treated ovaries.

Experiment III. In this experiment, 18 rabbits were used to determine the effects of transient ovarian exposure to cAMP on oocyte maturation. One ovary was perfused with $(\mathrm{Bu})_{2} \mathrm{CAMP}$ at a concentration of $10^{-5}, 10^{-4}$ or $10^{-3} \mathrm{M}$ for the first $2 \mathrm{~h}$. The contralateral ovary of each rabbit, which served as a control, was simultaneously perfused with medium alone. At $2 \mathrm{~h}$ after the onset of perfusion, both perfused ovaries were washed, transferred to new perfusate systems, and perfused with medium alone for an additional 10-h period (two-step perfusion). Perfusate samples were also obtained at the same time intervals as in Exp. II.

Radioimmunoassays. Progesterone was measured using a standard RIA kit (Diagnostic Products Corporation, Los Angeles, CA, USA), in which the progesterone antibody is bound covalently to the inner surface of polypropylene assay tubes (Kubasik et al., 1984; Yoshimura et al., 1986). The concentrations of the standard assay range from 0.1 to $40 \mathrm{ng} / \mathrm{ml}$. The interassay and intra-assay variations were $6 \cdot 8 \%$ and $2 \cdot 3 \%$ respectively.

Statistical analysis. Data for the maturity of cumulus-oocyte complexes and the concentrations of progesterone in Exp. I are presented as the mean \pm standard error. Statistical analysis of the progesterone data was performed using Student's $t$ test. The $\chi^{2}$ test with Yates' correction was used to assess percentage degeneration and percentage GVBD of follicular oocytes in Exps II and III. 


\section{Results}

\section{Experiment I}

Of the oocytes in cumulus cells incubated in the absence of $(\mathrm{Bu})_{2} \mathrm{cAMP}, 68 \%$ exhibited GVBD, indicating meiotic resumption, within the initial $4 \mathrm{~h}$ of incubation. After $6 \mathrm{~h}$ of incubation, most of the oocytes cultured in control medium $(95.2 \%)$ displayed evidence of meiotic resumption with GVBD. In contrast, $(\mathrm{Bu})_{2} \mathrm{cAMP}$ inhibited spontaneous oocyte maturation in a dose-related manner (Fig. 1). At 4 and $6 \mathrm{~h}$, this inhibition was significant at a concentration of $10^{-3} \mathrm{M}-(\mathrm{Bu})_{2} \mathrm{CAMP}$. As the time of incubation increased, $(\mathrm{Bu})_{2} \mathrm{cAMP}$ had less effect. By $12 \mathrm{~h}$ of incubation, $84 \cdot 2 \%$ of the oocytes had resumed meiosis despite the presence of $10^{-3} \mathrm{M}-(\mathrm{Bu})_{2} \mathrm{cAMP}$, and there was no difference between control groups and those exposed to $(\mathrm{Bu})_{2} \mathrm{cAMP}$.
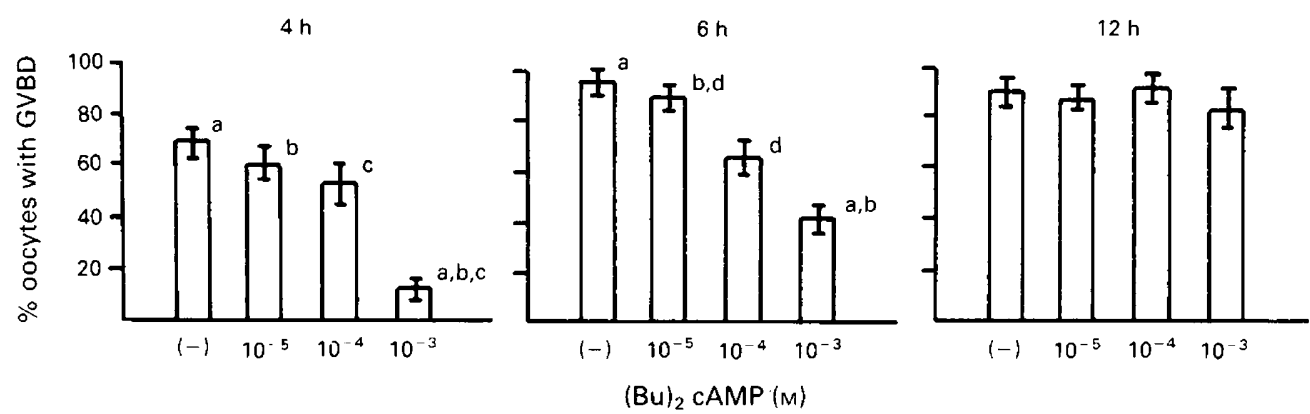

Fig. 1. Effect of $(\mathrm{Bu})_{2} \mathrm{cAMP}$ on spontaneous maturation of rabbit cumulus-oocyte complexes.

Data are expressed as the mean \pm s.e. of at least 6 different experiments containing a total of 20-30 complexes. Values with similar letters are significantly different: ${ }^{\mathrm{a} . \mathrm{b}} P<0.005,{ }^{\mathrm{c}} P<0.02$, ${ }^{\mathrm{d}} P<0.05$.

\section{Experiment II}

Perfusion of rabbit ovaries with $10^{-3} \mathrm{M}-(\mathrm{Bu})_{2} \mathrm{cAMP}$ did not induce ovulation or maturation of follicular oocytes in the absence of gonadotrophin (GVBD 17.1\% in those treated with $(\mathrm{Bu})_{2} \mathrm{cAMP}, 12.9 \%$ in controls). Although the percentage of follicular oocytes showing evidence of degeneration was increased in $(\mathrm{Bu})_{2}$ cAMP-treated ovaries $(31.4 \%)$ as compared with control ovaries $(25.8 \%)$, this difference was not significant.

\section{Experiment III}

Ovulation failed to occur during the 12 -h perfusion period (Table 1). $(\mathrm{Bu})_{2}$ cAMP at concentrations of $10^{-3}, 10^{-4}$ or $10^{-5} \mathrm{M}$ significantly increased maturation of follicular oocytes (Fig. 2). Maturation appeared to be dose-related. Although the percentage of follicular oocytes exhibiting degeneration was increased by exposure to all concentrations of $(\mathrm{Bu})_{2} \mathrm{cAMP}$, these increases were not significant (Table 1).

\section{Progesterone concentrations}

Progesterone concentrations in the effluent of ovaries perfused with or without hCG or $(\mathrm{Bu})_{2} \mathrm{cAMP}$ are illustrated in Fig. 3. In hCG-treated ovaries, progesterone concentration reached 
Table 1. Follicular oocyte characteristics from ovaries perfused with or without $(\mathrm{Bu})_{2} \mathrm{cAMP}$ for $2 \mathrm{~h}$

\begin{tabular}{lcccc}
\hline & \multicolumn{3}{c}{$(\mathrm{Bu})_{2} \mathrm{cAMP}$} & \\
\cline { 2 - 4 } & $10^{-3} \mathrm{M}$ & $10^{-4} \mathrm{M}$ & $10^{-5} \mathrm{M}$ & Control \\
\hline No. of ovaries perfused & 6 & 6 & 6 & 18 \\
No. of ovaries ovulating & 0 & 0 & 0 & 0 \\
No. of follicular oocytes & 43 & 42 & 38 & 119 \\
No. of oocytes showing: & & & & \\
$\quad$ GV & $12(1)^{*}$ & $15(4)$ & $23(2)$ & $104(12)$ \\
GVBD & $5(1)$ & $5(1)$ & $5(0)$ & $5(0)$ \\
M I & $14(4)$ & $13(1)$ & $6(2)$ & $6(4)$ \\
M II & $12(5)$ & $9(5)$ & $4(1)$ & $4(1)$ \\
\% Degeneration & $25.6 \%$ & $26.2 \%$ & $21 \cdot 1 \%$ & $14.3 \%$ \\
\hline
\end{tabular}

*Number of degenerated ova in parentheses.

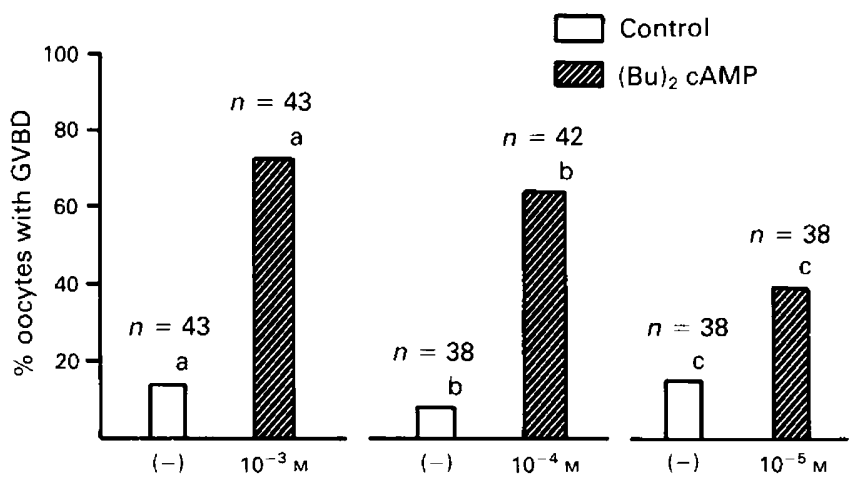

Fig. 2. Percentage of follicular oocytes achieving GVBD after the two-step perfusion in Exp. III. Ovaries were perfused with medium alone (control) or $(B u)_{2}$ cAMP for the first $2 \mathrm{~h}$. All ovaries were then transferred to medium alone for an additional $10 \mathrm{~h}$ of perfusion. Data are the mean of 6 different ovaries from at least 3 experiments. The s.e. was $\pm 4-6 \%$ for controls, and $\pm 7 \%, 9 \%$ and $14 \%$ for $10^{-3}, 10^{-4}$ and $10^{-5} \mathrm{M}-(\mathrm{Bu})_{2} \mathrm{cAMP}$ respectively. Values with similar letters are significantly different: ${ }^{\mathrm{a}, \mathrm{b}} P<0.001,{ }^{\mathrm{c}} P<0.05$.

its maximum $(140 \mathrm{ng} / \mathrm{ml}) 2 \mathrm{~h}$ after $\mathrm{hCG}$ administration and then declined. During continuous exposure of rabbit ovaries to $(\mathrm{Bu})_{2} \mathrm{cAMP}(\operatorname{Exp}$. II), progesterone values in the perfusate increased in linear fashion until $6 \mathrm{~h}$ after $(\mathrm{Bu})_{2} \mathrm{cAMP}$ administration. During the two-step perfusion (Exp. III), progesterone concentrations in the perfusate rose during the $2-\mathrm{h}$ exposure to $(\mathrm{Bu})_{2} \mathrm{cAMP}$, and then fell abruptly to values similar to those seen with medium alone when ovaries were transferred to medium without $(\mathrm{Bu})_{2} \mathrm{cAMP}$.

\section{Discussion}

The present study demonstrates that $10^{-3} \mathrm{M}-(\mathrm{Bu})_{2} \mathrm{cAMP}$ significantly inhibits spontaneous maturation of rabbit cumulus-oocyte complexes cultured in vitro for up to $6 \mathrm{~h}$. However, with increasing duration of incubation the majority of oocytes achieve GVBD. Cyclic AMP has been 


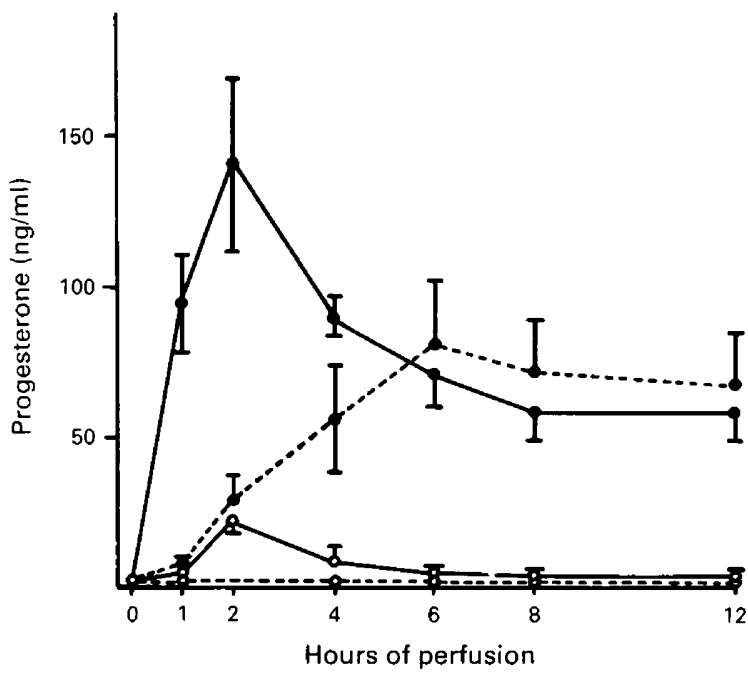

Fig. 3. Concentrations of progesterone in the medium of perfused rabbit ovaries: 12-h perfusions were done in the presence of $50 \mathrm{i} . \mathrm{u}$. hCG $(-0), 10^{-3} \mathrm{M}-(\mathrm{Bu})_{2} \mathrm{cAMP}(---)$, or medium alone $(\mathrm{O}---\mathrm{O})$. One group of ovaries was exposed to $10^{-3} \mathrm{M}-(\mathrm{Bu})_{2} \mathrm{cAMP}$ for $2 \mathrm{~h}$ and then transferred to medium alone for an additional $10 \mathrm{~h}$ of perfusion $(\mathrm{O}-\mathrm{O})$. Values are mean \pm s.e. with $n=6$ per treatment.

proposed as the physiological intrafollicular inhibitor of oocyte maturation. The inhibitory effect of cAMP derivatives on isolated oocytes is reversible and can be overcome by LH (Dekel \& Beers, 1978). Our results suggest that cAMP may play a significant role in the intrafollicular arrest of oocyte meiosis in rabbits as it does in other mammalian species (Cho et al., 1974; Dekel \& Beers, 1978).

In both the hamster and rat (Sridharan et al., 1974; Baranczuk \& Fainstat, 1976) progesterone appears to play a role in ovulation. However, in the in-vitro perfused rabbit ovary, progesterone does not influence the process of ovulation or maturation (Hamada et al., 1979; Holmes et al., 1985; Yoshimura et al., 1986, 1987). The data provide additional evidence that the marked elevation in progesterone concentrations produced by gonadotrophin or cAMP exposure does not induce follicle rupture or oocyte maturation.

Holmes et al. (1986) have demonstrated that the inclusion of forskolin in a similar rabbit ovarian perfusion system induces ovulation in the absence of gonadotrophins. Similar observations have been made in a rat perfusion system (Brannström et al., 1987). Since forskolin activates adenylate cyclase (Seamon et al., 1981), Holmes et al. (1986) suggest that specific compartmental release of cAMP may be capable of initiating ovulation. Although in those studies the release of cAMP was greater after forskolin treatment than after $\mathrm{LH}$, the ovulatory efficiency was lower in the forskolin group. In contrast, the present study shows that continuous or transient exposure to $(\mathrm{Bu})_{2}$ cAMP failed to induce ovulation in the absence of gonadotrophin. While it is possible that cAMP is not an essential component of the ovulatory process, it is also possible, as suggested by Holmes et al. (1986), that the location of the release of cAMP within the cell is critical. Binding of gonadotrophin to its receptor may release cAMP at a location that stimulates specific protein kinases critical for follicle rupture. Perfusion with $(\mathrm{Bu})_{2} \mathrm{cAMP}$ may not achieve the compartmental concentration of cAMP sufficient to activate these kinases and activation with forskolin may be less than that with gonadotrophins.

Although gonadotrophins stimulate steroidogenesis through an increase in ovarian cAMP (Marsh, 1976), the pattern of progesterone production by the perfused rabbit ovary after exposure 
to hCG differed from that after $(\mathrm{Bu})_{2} \mathrm{cAMP}$. This discrepancy may relate to differences in compartmentalization between endogenous cAMP and the exogenous dibutyryl derivatives, or may represent differences in concentration of cAMP achieved with the two procedures.

Preovulatory rabbit follicles respond to gonadotrophin administration with increased cAMP formation (Marsh et al., 1973; Patwardhan \& Lanthier, 1984). The increase in follicular cAMP concentrations within $0.5 \mathrm{~h}$ was followed by rapid decline at $3-4 \mathrm{~h}$ after hCG stimulation. These results suggest that continuous exposure to the exogenous nucleotide may not necessarily simulate physiological conditions. The transient exposure to $(\mathrm{Bu})_{2} \mathrm{cAMP}$ in the two-step perfusion experiments, which attempts to reproduce the physiological pattern, significantly accelerated meiotic resumption, while continuous exposure to $(\mathrm{Bu})_{2} \mathrm{cAMP}$ maintained oocytes in meiotic arrest. These data are consistent with other reports that cAMP may be involved in the intrafollicular inhibition of oocyte maturation (Cho et al., 1974; Magnusson \& Hillensjo, 1977; Dekel \& Beers, 1978, 1980; Racowsky, 1983).

The results of these experiments may be applied to resolve the paradoxical situation in which cAMP significantly inhibits spontaneous maturation of cumulus-oocyte complexes, while inducing meiotic resumption in intrafollicular oocytes following the preovulatory gonadotrophin surge. Continuous exposure to elevated cAMP exerts an inhibitory effect on oocyte maturation in isolated cumulus-oocyte complexes and intrafollicular oocytes. In contrast, transient exposure of preovulatory follicles to elevated cAMP accelerates the resumption of meiosis in a dose-dependent manner. These data suggest that the alterations of cAMP levels achieved in response to gonadotrophin stimulation may serve as an early signal in the sequence of events leading to resumption of oocyte meiosis.

We thank Fran Karas for expert assistance in the preparation of the manuscript. The work was supported by NIH grant HD-19430 and the Rockefeller, Connelly and Mitchell and Lillian Duberstein Foundations.

\section{References}

Baranczuk, R.J. \& Fainstat, T. (1976) Progesteroneinduced ovulation of the hamster ovary in vitro. $J$. Endocr. 70, 317-318.

Brackett, B.G. \& Oliphant, G. (1975) Capacitation of rabbit spermatozoa in vitro. Biol. Reprod. 12 , 260-274.

Brannström, M., Koos, R.D., LeMaire, W.J. \& Janson, P.O. (1987) Cyclic adenosine 3',5'-monophosphateinduced ovulation in the prefused rat ovary and its mediation by prostaglandins. Biol. Reprod. 37, 1047-1053.

Cho, W.K., Stern, S. \& Biggers, J.D. (1974) Inhibitory effect of dibutyryl cAMP on mouse oocyte maturation in vitro. J. exp. Zool. 187, 383-386.

Dekel, N. \& Beers, W.H. (1978) Rat oocyte maturation in vitro: relief of cyclic AMP inhibition by gonadotropins. Proc. natn. Acad. Sci. U.S.A. 75, 4369-4373.

Dekel, N. \& Beers, W.H. (1980) Development of the rat oocyte in vitro: inhibition and induction of maturation in the presence or absence of the cumulus oophorus. Devl Biol. 75, 247-254.

Hamada, Y., Wright, K.H. \& Wallach, E.E. (1979) The effects of progesterone and human chorionic gonadotropin on ovulation in the in vitro perfused rabbit ovary. Fert. Steril. 32, 335-339.

Holmes, P.V., Sogn, J., Schillinger, E. \& Janson, P.O. (1985) Effects of high and low preovulatory con- centrations of progesterone on ovulation from the isolated perfused rabbit ovary. J. Reprod. Fert. 75, 393-399.

Holmes, P.V., Hedin, L. \& Janson, P.O. (1986) The role of cyclic adenosine $3^{\prime}, 5^{\prime}$-monophosphate in the ovulatory process of the in vitro perfused rabbit ovary. Endocrinology 118, 2195-2202.

Kobayashi, Y., Wright, K.H., Santulli, R. \& Wallach, E.E. (1981) Ovulation and ovum maturation in the rabbit ovary perfused in vitro. Biol. Reprod. 24, 483-490.

Kubasik, N.P., Hallauer, G.D. \& Brodows, R.G. (1984) Evaluation of a direct solid-phase radioimmunoassay for progesterone, useful for monitoring luteal function. Clin. Chem. 30, 284286.

Lambertsen, C.J., Jr, Greenbaum, D.F., Wright, K.H. \& Wallach, E.E. (1976) In vitro studies of ovulation in the perfused rabbit ovary. Fert. Steril. 27, 178-187.

Liberman, M.E., Tsafriri, A., Bauminger, S., Collins, W.P., Ahren, K. \& Lindner, H.R. (1976) Oocyte meiosis in cultured rat follicles during inhibition of steroidogenesis. Acta endocr., Copenh. 83, 151-157.

Magnusson, C. \& Hillensjo, T. (1977) Inhibition of maturation and metabolism in rat oocytes by cyclic AMP. J. exp. Zool. 201, 139-147.

Marsh, J.M. (1976) The role of cyclic AMP in gonadal steroidogenesis. Biol. Reprod. 14, 30-53. 
Marsh, J.M., Mills, T.M. \& LeMaire, W.J. (1973) Preovulatory changes in the synthesis of cyclic AMP by rabbit Graafian follicles. Biochim. Biophys. Acta 304, 197-202.

Patwardhan, V.V. \& Lanthier, A. (1984) Concentration of cyclic AMP in rabbit ovaries in the preovulatory period after administration of luteinizing hormone or human chorionic gonadotropin. Acta endocr., Copenh. 106, 260-264.

Pincus, G. \& Enzmann, E.V. (1935) The comparative behavior of mammalian eggs in vivo and in vitro. I. The activation of ovarian eggs. J. exp. Med. 62, 665-675.

Racowsky, C. (1983) Androgenic modulation of cyclic adenosine monophosphate (cAMP)-dependent meiotic arrest. Biol. Reprod. 28, 774-787.

Seamon, K.B., Padgett, W. \& Daly, J.W. (1981) Forskolin: unique diterpene activator of adenylate cyclase in membranes and intact cells. Proc. natn. Acad. Sci. U.S.A. 78, 3363-3367.

Sridharan, B.N., Meyer, R.K. \& Karavolas, H.J. (1974) Induction of ovulation by pregn-5-ene-3,20-dione and progesterone in immature rats treated with PMSG and phenobarbital. J. Reprod. Fert. 39, 259-266.

Thibault, C. (1977) Are follicular maturation and oocyte maturation independent processes? J. Reprod. Fert. 51, 1-15.

Yoshimura, Y., Kitai, H., Santulli, R., Wright, K. \& Wallach, E.E. (1985) Direct ovarian effect of clomiphene citrate in the rabbit. Fert. Steril. 43, 471-476.

Yoshimura, Y., Hosoi, Y., Atlas, S.J., Bongiovanni, A.M. \& Wallach, E.E. (1986) The effects of ovarian steroidogenesis on ovulation and fertilizability in the in vitro perfused rabbit ovary. Biol. Reprod. 35, 943-948.

Yoshimura, Y., Hosoi, Y., Bongiovanni, A.M., Santulli, R., Atlas, S.J. \& Wallach, E.E. (1987) Are ovarian steroids required for ovum maturation and fertilization? Effects of cyanoketone on the in vitro perfused rabbit ovary. Endocrinology 120, 2555-2561.

Received 6 May 1988 